\title{
A plasmonic chip for biomarker discovery and diagnosis of type 1 diabetes
}

\author{
Bo Zhang ${ }^{1,2,5}$, Rajiv B Kumar ${ }^{1,3,5}$, Hongjie Dai ${ }^{1,2}$, and Brian J Feldman ${ }^{1,3,4}$ \\ ${ }^{1}$ Stanford University, Stanford, California, USA \\ ${ }^{2}$ Department of Chemistry, Stanford University School of Humanities and Sciences, Stanford, \\ California, USA \\ ${ }^{3}$ Department of Pediatrics, Stanford University School of Medicine, Stanford, California, USA \\ ${ }^{4}$ Program in Regenerative Medicine, Stanford University, Stanford, California, USA
}

\section{Abstract}

Type 1 diabetes (T1D) is an autoimmune disease, whereas type 2 diabetes (T2D) results from insulin resistance and beta cell dysfunction. Previously, the onset of these two separate diseases was easily distinguished, with children being most at risk for T1D and T2D occurring in overweight adults. However, the dramatic rise in obesity, coupled with the notable increase in T1D, has created a large overlap in these previously discrete patient populations. Delayed diagnosis of T1D can result in severe illness or death, and rapid diagnosis of T1D is critical for the efficacy of emerging therapies. However, attempts to apply next-generation platforms have been unsuccessful for detecting diabetes biomarkers. Here we describe the development of a plasmonic gold chip for near-infrared fluorescence-enhanced (NIR-FE) detection of islet cell-targeting autoantibodies. We demonstrate that this platform has high sensitivity and specificity for the diagnosis of T1D and can be used to discover previously unknown biomarkers of T1D.

The World Health Organization projects that the global prevalence of diabetes will increase from 217 million cases in 2005 to 366 million by 2030 (ref. 1). Diabetes remains a widespread disease in adults and is now the second most common chronic disease in children. For unclear reasons, the rate of T1D, or autoimmune diabetes, in children is rising by $3 \%$ annually ${ }^{2-4}$, with a projected $70 \%$ increase in prevalence between 2004 and 2020 (ref. 5). Although T1D was once considered a pediatric disease, approximately one-fourth of affected individuals are now diagnosed during adulthood ${ }^{6}$. Concurrently, the rate of T2D, also called metabolic diabetes, has escalated exponentially in children since the early 1990s,

\footnotetext{
Reprints and permissions information is available online at http://www.nature.com/reprints/index.html. Correspondence should be addressed to B.J.F. (feldman@ stanford.edu) or H.D. (hdai1@ stanford.edu). ${ }^{5}$ These authors contributed equally to this work.

Any Supplementary Information and Source Data files are available in the online version of the paper.

B.J.F. conceived of the study. B.Z., R.B.K., H.D. and B.J.F. designed the experiments, analyzed the data and wrote the manuscript. B.Z. and R.B.K. conducted the experiments.
} 
related in part to the global obesity epidemic ${ }^{1,4,7,8}$. As recently as 20 years ago, the probability of a young child developing T2D, or an overweight adult developing T1D, was low enough that diagnostic and treatment decisions were initiated on the basis of epidemiologic assumptions. However, the simultaneous rise in incidence of T1D and $\mathrm{T} 2 \mathrm{D}^{9,10}$ has resulted in considerable overlap in the patient populations and a paradigm shift for these previously easily distinguishable diseases. A fundamental problem in current diabetes care is that diagnostic tests are slow and costly, delaying definitive diagnosis and making this key test inaccessible to regions of the globe with limited resources.

In addition to the fact that present platforms fail to meet current diagnostic needs, arising T1D therapies are critically dependent on faster diagnostics than these existing platforms can provide. Highly encouraging preliminary studies indicate that immune modulation and antigen-specific therapies fundamentally alter the natural history of $\mathrm{T}^{1} \mathrm{D}^{11,12}$. These breakthrough therapies have the potential to protect pancreatic beta cell function as they move toward broad implementation in the patient population. However, early results from these studies indicate that therapeutic interventions are most effective when administered temporally close to the time of diagnosis, with delays as short as days to weeks potentially limiting efficacy ${ }^{12,13}$. The current platforms for diagnosing diabetes are unable to meet this important emerging requirement and could prevent patients with T1D from receiving the maximum benefit from their therapy. In spite of the clear medical need, developing nextgeneration diagnostic tests has been unexpectedly challenging. Over the past two decades, many groups have demonstrated that the usual platforms, including lateral flow assays and ELISA, underperform when applied to T1D diagnostics, failing to meet the necessary sensitivity and specificity demands of this clinical situation ${ }^{14,15}$.

The detection of autoantibodies against one or more pancreatic islet antigens (insulin, glutamic acid decarboxylase (GAD65) and/or tyrosine phosphatase islet antigen 2 (IA2 or ICA512)) in a patient with hyperglycemia is diagnostic of T1D and distinguishes the disease from $\mathrm{T} 2 \mathrm{D}^{16}$. Screening for autoimmunity to islet cells in T1D sera was first described four decades ago using an indirect immunofluorescence assay on frozen sections of human pancreata $^{17}$. Methodology has progressed with the identification of the three primary islet antigens ${ }^{16}$, and numerous international workshops have been conducted with a focus on improving T1D autoantibody measurements ${ }^{18,19}$.

Autoantibodies against insulin are generally the first to appear in individuals with T1D and are classically the most difficult to detect ${ }^{20}$. Although it is not fully understood why detection of insulin-specific autoantibodies has been recalcitrant to standard approaches, the challenge has been attributed to the loss of critical tertiary structure in epitope binding sites when insulin is immobilized in solid-phase platforms ${ }^{21}$. Improved relative sensitivity has been achieved using radioimmunoassay (RIA) with target antigens in fluid phase ${ }^{14}$. However, the Diabetes Antibody Standardization Program demonstrated that RIA had poor overall performance and low sensitivity for detection of insulin-specific autoantibodies ${ }^{20,22}$. This method remains the de facto standard for detection of autoantibody biomarkers of T1D, and there is currently no validated nonradioactive assay available ${ }^{21}$. Importantly, this method is time and labor intensive and is unable to provide results before a physician needs to initiate therapy, thus limiting the therapeutic options available to the patient. 
Using recent advances in plasmonic gold substrate for NIR-FE detection ${ }^{23,24}$, we developed a platform that overcomes the major challenges to rapid, sensitive and specific diagnosis of diabetes. This approach allows successful detection of T1D autoantibodies in ultralow volumes of human serum, such as that obtained from a finger-prick sample, with results available after a short processing time. We found that our platform has sensitivity and specificity equal to those of RIA. In addition, this new assay permits multiplexing of the islet antigens, provides autoantibody signal quantification, detects several isotypes of autoantibodies via multicolor fluorescence labeling on a single chip, is inexpensive and, notably, provides a point-of-care platform.

\section{RESULTS}

\section{Gold plasmonic chip enables detection of islet-targeting autoantibodies}

To address the medical need for a rapid, sensitive and specific diagnostic test for diabetes, we first examined several platforms frequently used in other similar clinical situations, including lateral flow assays and ELISA. Consistent with what has been reported by others ${ }^{14,21,25}$, we were unable to achieve the needed sensitivity and specificity requirements using these standard platforms to detect islet antigen-specific autoantibodies (Supplementary Figs. 1 and 2 and Supplementary Table 1).

We next applied nanotechnology to overcome the major obstacles of low signal intensity and poor sensitivity that we found using other approaches. We generated multiplexed islet antigen microarrays using nanostructured gold islands on a glass surface (Fig. 1a). The gold islands ensure the generation of abundant nanogaps ( 10-nm range) that support electric field enhancement and surface plasmonic resonance to improve NIR-FE detection by $\sim 100$ fold $^{23}$ (Fig. 1a). In addition, we applied a biocompatible branched polyethylene glycol (PEG) layer over the gold islands to help preserve the native conformation of the islet antigens ${ }^{26}$ (Fig. 1b). To form a sandwich assay on this platform for islet-specific autoantibody quantification, we immobilized target islet antigen on the plasmonic chip, probed it with human serum (to capture specific autoantibodies against the target antigen) and then detected it with IRDye800-labeled anti-human IgG antibody. The abundance of specific autoantibody in the serum was reflected by the measured intensity of IRDye 800 fluorescence (Fig. 1c). A calibration curve for insulin-, GAD65- or IA2-specific autoantibodies was first obtained on a plasmonic chip using commercial reference samples containing known concentrations of the autoantibody. We observed a sensitivity increase of two to three orders of magnitude on the gold substrate compared with on glass. Notably, we obtained calibration titration curves exhibiting $<10 \%$ coefficient variation with different batches of plasmonic chips in independent experiments even at low concentrations of autoantibodies, down to $0.1 \mathrm{U} / \mathrm{ml}$ (Fig. 1c), demonstrating the high reproducibility and consistency of the plasmonic gold substrates. In contrast to the plasmonic gold substrates, vacuum-evaporated smooth gold films produced a fluorescence quenching, instead of enhancing, effect, resulting in lower sensitivity than traditional glass substrate (Fig. 1c). Other microarray substrates, such as glass, nitrocellulose or smooth gold, had much lower sensitivity and specificity than the plasmonic gold surface (Supplementary Figs. 2 and 3). 
Notably, the plasmonic gold platform had better sensitivity than RIA for detecting insulinspecific autoantibodies (Fig. 1d).

\section{Detection of islet antigen-specific autoantibodies in ultralow volumes}

In addition to improved sensitivity, the use of this plasmonic chip for diabetes diagnostics has several other advantages over RIA (Supplementary Table 2), including the ability to use ultralow sample volumes. RIA testing requires several milliliters of blood, making it necessary for patients to go to a phlebotomy laboratory. However, the most rapid and versatile way to confirm a diagnosis is at the point of care, such as a primary care physician's office. When the required volume of blood is ultralow, a sample may be obtained from a finger prick rather than requiring a phlebotomy laboratory. This capability would fundamentally alter the current diagnostic approach.

We constructed a plasmonic chip with a six-plexed antigen array for simultaneous multiple islet antigen-specific autoantibody detection with low sample volumes (Fig. 2a). The islet antigens were each robotically printed in triplicate spots onto the branched PEG-coated gold film, resulting in a microarray with feature diameters of $\sim 400 \mu \mathrm{m}$. As a technical positive control, we printed human IgG for recognition by the secondary detection antibody that we used. As a biological positive control, we printed tetanus toxoid, as immunization to tetanus is part of standard health care practice in the United States. Spots of PBS were used as negative controls.

To validate our platform using ultralow sample volumes, we tested volunteer patients with new-onset diabetes who were simultaneously undergoing standard RIA testing for islet antigen-specific autoantibodies. About $2 \mu \mathrm{l}$ of human serum or blood was diluted 1:10 and used to probe the chips (Online Methods and Supplementary Fig. 4). After processing (Online Methods), we analyzed the chips in a microarray scanner to detect and quantify the signals. We detected positive signals on the IgG and tetanus spots and no signal on the PBS spots on every chip tested (Fig. 2a). If a patient's serum contained autoantibodies against one or more islet antigens, we detected a positive signal specifically on the respective antigen spots (Fig. 2b). With this technique, we also were able to detect T1D autoantibodies in ultralow volumes of whole-blood samples from humans (Fig. 2c). The outcome of autoantibody detection in whole-blood samples was consistent with that in processed serum samples from the same patient (Fig. 2c). The extremely small volume of blood required for our assay indicates that it will be feasible to use finger-prick sampling rather than venous blood draws (Fig. 2d).

\section{Plasmonic chip and RIA have the same sensitivity and specificity}

We collected samples from individuals with new-onset diabetes and nondiabetic controls at Stanford University Medical Center (Supplementary Table 3). We excluded subjects for whom informed consent was not granted or not possible, and those known to have conditions such as active bleeding, severe anemia or other medical conditions whereby removal of an extra tube of blood would increase risk to the subject. In addition, we excluded any subject treated with insulin for more than $3 \mathrm{~d}$, as exogenous insulin injected subcutaneously is thought to result in nonpathologic antibody production against this 
protein ${ }^{27}$. We collected samples from 39 patients with new-onset diabetes (26 were later diagnosed with T1D and 13 with T2D) and 5 nondiabetic controls.

To establish positive signal intensity cutoffs on the plasmonic chip, we tested spiked serum control samples containing autoantibodies at the cutoff concentration to determine their mean fluorescence intensity (MFI) equivalence on our platform (Fig. 1c). To facilitate direct comparison of the described assay with the currently used standard, we tested an aliquot of the same blood samples used on the plasmonic gold chip using RIA in a commercial laboratory for every diabetic subject. The results of both assays were compared to the ultimate clinical diagnosis determined by disease progression and insulin requirement 6 months after the initial presentation (Online Methods). One subject with T1D had GAD65specific autoantibody testing performed on a different RIA platform, and one subject with T2D had insulin-specific and GAD65-specific autoantibody testing performed on a different RIA platform; these values were not included for statistical analysis, and omitting these values did not improve the results. As expected, all of the nondiabetic controls tested negative for all three autoantibodies on the plasmonic gold chip platform (Fig. 3 and Supplementary Fig. 5). We tested samples from all the patients with diabetes for each autoantibody three different times, on different days, using different plasmonic chips, with excellent reproducibility (Supplementary Fig. 6). The mean titers for each autoantibody for every patient are plotted on the same graph in Figure 3 and individual autoantibody graphs in Supplementary Figure 5. We found that the plasmonic gold chip and RIA both have a sensitivity of $100 \%$ and specificity of $85 \%$ in the patients tested (Table 1). We also evaluated the same serum samples tested on the plasmonic gold chip using microarrays formed on glass and nitrocellulose. We found that the NIR fluorescence signal-to-noise ratios for the detection of islet antigen-specific autoantibodies were far superior on the plasmonic platform (Supplementary Fig. 2 and Supplementary Table 1).

\section{Multiplexed detection of potentially new and early biomarkers}

Although modern RIAs favor IgG detection in their protein A immunoprecipitation step, they do not distinguish among immunoglobulin isotypes ${ }^{28,29}$. IgM antibodies are produced early in an immune response and are later replaced by isotype switching to IgG antibodies ${ }^{30}$. Taking advantage of our ability to enhance the fluorescence of multiple NIR dyes (e.g., Cy3, Cy5 and IRDye800 by 3-, 50- and 100-fold, respectively (Supplementary Fig. 3)) with non-overlapping emission spectra (Fig. 4a), we achieved simultaneous detection of three subclasses of human autoantibodies (IgG, $\operatorname{IgM}$ and $\operatorname{IgA}$ ) against each islet antigen (Fig. 4b). This was done by using Cy3-labeled anti-human IgG secondary antibody, Cy5-labeled antihuman IgM secondary antibody and IRDye800-labeled anti-human IgA secondary antibody to probe the microarray spots of captured human IgG, IgM and IgA autoantibodies from a patient's serum. Of note, we chose the dye with the lowest NIR fluorescent enhancement for reporting the antibody subtype in the highest abundance in human serum. This was the first time multicolor microarrays were developed on a plasmonic substrate. Using this multicolor detection scheme, we identified a patient with new-onset diabetes who tested positive for IgM autoantibodies against insulin and GAD65 and positive for IgG autoantibodies against GAD65 and IA2 (Fig. 4c). Although further research is needed to elucidate whether testing for IgM (or IgA) autoantibodies is clinically useful, this discovery exemplifies that the 
platform can be used as a new research tool that, unlike currently used panels, enables the testing of an enriched set of potential biomarkers.

\section{DISCUSSION}

For over two decades, it has been recognized that a rapid and globally accessible highsensitivity diagnostic test for diabetes would substantially advance the field by allowing prompt diagnosis of patients that require T1D therapy and distinguishing the disease from T2D. In more recent years, this need has increased as it appears that expeditious intervention with next-generation T1D therapies affects the efficacy of these emerging treatments. In spite of this tremendous need, developing the technology has proved considerably more challenging than many other disease diagnostic platforms.

T1D is defined by the detection of one or more islet antigen-specific autoantibodies. However, these biomarkers are difficult to identify and quantify with sufficient signal in human blood. When present, these antibodies are found at concentrations in the picomolar to femtomolar range, and it is challenging to differentiate their signals from other antibodies and blood components. We, and others, have demonstrated that traditional protein assays that use optical reporters, including ELISA and protein microarrays on nitrocellulose or glass substrates, fail to reliably detect diabetes autoantibodies. The low signal and high background noise when these assays are used to measure islet cell-targeting autoantibodies is a likely cause of these failures (Supplementary Fig. 2). This is further confounded by the inherent challenge of maintaining the native structure of the insulin epitope-autoantibody binding sites when immobilizing this important antigen on a solid surface ${ }^{21}$ (Supplementary Fig. 7). The unique surface chemistry of the plasmonic gold chip platform permits immobilization of islet antigens while preserving antibody binding. In addition, this surface chemistry maximizes analyte capture while resisting nonspecific binding, resulting in low background, and has a diagnostic sensitivity equivalent to that of RIA. Furthermore, the plasmonic gold chips have a batch-to-batch coefficient variation $<10 \%$ at low analyte concentrations (down to $0.1 \mathrm{U} / \mathrm{ml}$ ) (Fig. 1c,d and Supplementary Fig. 6). NIR fluorescence enhancement on plasmonic gold films emanates from local electric field enhancement by the abundant nanoscale gaps between gold nanoislands and enhanced radiative decay rates or fluorescence quantum yield due to resonance emission dipole coupling to plasmonic modes ${ }^{31}$. The NIR fluorescence enhancement of positive signals, combined with low, nonspecific background signals, are responsible for the broader dynamic range and lower limit of detection versus conventional substrates, leading to an improved sensitivity in T1D diagnosis (Fig. 1c,d and Supplementary Table 1). To our knowledge, this is the first time that protein microarrays on plasmonic gold chips have been used for human disease diagnosis.

Furthermore, the ability to perform isotype-specific analysis enabled our identification of IgM and IgA autoantibodies. We are not aware of any previous report of multiplexed detection of these isotypes in a single assay platform for a patient with diabetes, demonstrating how this platform can be used as a new research tool to test for putative previously unknown biomarkers. Identifying biomarkers that can detect acute islet cell 
injury earlier than those currently used would greatly advance the field by facilitating an array of basic and clinical research opportunities.

Although RIA is the current standard for detecting T1D autoantibodies, this method is cumbersome and not readily available in some clinical settings. In addition to time intensity, other disadvantages of RIA include radioisotope requirement, cost, the inability to multiplex autoantibody detection and the relatively large amounts of serum required, necessitating venipuncture. Though ELISA is the standard for protein quantification and is widely used for research and clinical purposes, it is inadequate for autoantibody detection in T1D because of high background signals caused by nonspecific binding of human antibodies and changes in tertiary protein structure. The plasmonic gold platform allows simultaneous detection and quantification of autoantibodies to islet antigens and their isotypes. It requires only a $2-\mu \mathrm{l}$ sample of whole blood without further processing to obtain serum. This can be acquired from a simple finger prick, which opens the possibility of point-of-care T1D diagnosis.

In addition to addressing the current clinical need for improved diabetes diagnostics, we believe that this technology will enable a broad range of advances in basic and clinical research that were not previously feasible. For example, serial monitoring of autoantibodies in patients undergoing new interventions might predict efficacy, as therapies that protect against continued islet cell destruction may correlate with a decline in autoantibody titer. Furthermore, following antibody levels at high resolution in patients considered high risk would yield great insight into the natural history of the development of diabetes. Ultimately, we believe this technology could be deployed to facilitate screening for islet antigenspecific autoantibodies, identifying those who would otherwise be at risk for progression to diabetic ketoacidosis and testing of preventative interventions prior to the onset of clinical symptoms.

\section{Methods}

Methods and any associated references are available in the online version of the paper.

\section{ONLINE METHODS}

\section{Materials}

Superfrost Plus glass slides were purchased from Fisher Scientific and rinsed with acetone, isopropanol and methanol prior to use. Two-pad and sixteen-pad Whatman FAST nitrocellulose slides, chloroauric acid trihydrate, hydroxylamine $\mathrm{HCl}$, sodium borohydride, cysteamine, mercaptohexadecanoic acid, 1-ethyl-3-(3-dimethylaminopropyl) carbodiimide and $N$-hydroxysuccinimide (NHS) were purchased from Sigma-Aldrich. Ammonium hydroxide (30\% ammonia) and Hyclone fetal bovine serum were purchased from Fisher Chemicals. IR800cw-NHS ester was purchased from Licor Biosciences. Six-armed PEGamine was purchased from SunBio, and fetal bovine serum was purchased from Invitrogen.

Recombinant human insulin was purchased from Lilly, GAD65 and IA2 (ICA512) antigens were purchased from Kronus and tetanus toxoid antigen was purchased from Santa Cruz Biotech. Goat anti-human IgG, IgM and IgA antibodies were purchased from Vector 
Laboratories (Catalog \# AI-3080, AI-3020 and AI-3030) and used at a final concentration of $1 \mathrm{nM}$.

\section{Human serum}

Approval for this study was obtained from the Stanford University Institutional Review Board. Once parental consent and subject assent (when appropriate) were obtained, $2.5 \mathrm{ml}$ of blood was drawn in a red-top venipuncture tube (silicon-coated glass) and $2.5 \mathrm{ml}$ in a lavender-top venipuncture tube (liquid $\mathrm{K}_{2}$ EDTA in glass). Samples were stored at $4{ }^{\circ} \mathrm{C}$ until processing. For processing, the $2.5 \mathrm{ml}$ of whole blood collected in the lavender-top tube was divided into $125-\mu \mathrm{l}$ aliquots in cryovials and stored at $-80{ }^{\circ} \mathrm{C}$. The $2.5 \mathrm{ml}$ collected in the red-top tube was centrifuged at 300 r.p.m. for $15 \mathrm{~min}$. The serum supernatant was then divided into $75-\mu \mathrm{l}$ aliquots in cryovials and stored at $-80{ }^{\circ} \mathrm{C}$. Samples were subsequently thawed and assessed in parallel.

\section{Clinical diagnosis and monitoring of patients with T2D}

All patients were monitored regularly for at least 6 months after diagnosis. Of the 13 patients categorized as T2D, 10 were transitioned completely off of insulin for over 1 month without developing ketosis or diabetic ketoacidosis and were able to maintain good blood glucose control with diet and exercise, or diet, exercise and metformin. The other three patients had prolonged periods of insulin omission without ketosis, and all three of these patients tested negative for autoantibodies on both platforms. One patient tested positive for a previously unknown mutation in the HNF4A gene (that has not been reported to be associated with maturity-onset diabetes of the young).

\section{ELISA procedure}

We coated 96-well microtiter plates (BD Falcon) with $100 \mu \mathrm{g}$ recombinant human insulin (Lilly). The antigen was incubated at $4{ }^{\circ} \mathrm{C}$ overnight followed by washing with PBS with Tween 20 (PBST) and then blocked overnight at $4{ }^{\circ} \mathrm{C}$ with $5 \%$ nonfat milk. We dispensed $100 \mu \mathrm{l}$ of 1:10 diluted serum for each subject into the wells and incubated them overnight at $4{ }^{\circ} \mathrm{C}$. Samples were incubated with a 1:5,000 dilution of rabbit anti-human IgG conjugated with horseradish peroxidase (Santa Cruz Biotechnology, SC-2769) for $2 \mathrm{~h}$ and then washed several times. Following plate washing, $50 \mu \mathrm{l}$ of 3,3',5,5'-tetramethylbenzidine (Sigma) was added to each well for $30 \mathrm{~min}$, and then an equal volume of $2 \mathrm{M} \mathrm{H}_{2} \mathrm{SO}_{4}$ (Sigma) was added to stop the substrate reaction. The optical density $(450 \mathrm{~nm})$ of each well was quantified using a plate reader (BioTek Synergy H1).

\section{Preparation of evaporated gold film on glass slides}

Evaporated gold films were deposited via e-beam evaporation on an Innotec ES26C E-Beam Evaporator. The vacuum pressure of the system prior to deposition was $5 \times 10^{-7}$ Torr. The e-beam parameters were $10 \mathrm{kV}$ at $\sim 0.1 \AA$, which resulted in a deposition rate of roughly 2 $\AA$ Às. 


\section{Preparation of plasmonic gold film on glass slides}

Glass slides were immersed in $3 \mathrm{mM} \mathrm{HAuCl}_{4}$ followed by addition of ammonium hydroxide at $20 \mu \mathrm{l}$ ammonium hydroxide per $\mathrm{ml}$ of $\mathrm{HAuCl}_{4}$ solution with rapid shaking for $1 \mathrm{~min}$. The slide was washed twice with deionized water to remove unbound gold ions and immersed into $1 \mathrm{mM} \mathrm{NaBH}_{4}$ to reduce gold clusters on the glass slide to gold nanoparticle seeds. After further washing twice with water, the slides were incubated in a solution of $\mathrm{HAuCl}_{4}$ and hydroxylamine at a 1:1 ratio and shaken for $5 \mathrm{~min}$, followed by a 10-min incubation to complete the growth step. After washing with water twice and drying, the slide was checked for plasmon resonance by using a Cary 300UV-Vis-NIR absorbance spectrometer after correcting for background absorbance from the glass substrate. Scanning electron micrographs were acquired on an FEI XL30 Sirion SEM with FEG source at 5-kV acceleration voltage.

\section{Construction of multilayer surface chemistry on gold film}

Gold slides were immersed into $10 \mathrm{mM}$ mercaptohexadecanoic acid in ethanol overnight at room temperature. After rinsing with ethanol and drying, the carboxylic groupfunctionalized gold slide was immersed in a solution of $20 \mu \mathrm{M}$ 6-arm PEG-amine (Mn $\sim 10,000 \mathrm{Da}$ ) and $20 \mathrm{mM}$ each of 1-ethyl-3-(3-dimethylaminopropyl) carbodiimide and NHS in dimethylformamide (DMF). After rinsing the gold slide with DMF and ethanol and then drying it, the substrate was immersed in $10 \mathrm{mM}$ succinic anhydride DMF solution with triethylamine at $1 \mu \mathrm{l} / \mathrm{ml}$. This step transforms the free amine groups on the PEG chain into carboxylic groups. Following another washing step with DMF and ethanol, the slide was incubated in $20 \mathrm{mM}$ each of 1-ethyl-3-(3-dimethylaminopropyl) carbodiimide and NHS in DMF to activate the carboxylic group.

\section{Microarray printing and microarray sandwich assay procedure}

The NHS-activated gold slides above (or nitrocellulose slides, glass slides or NHS-activated evaporated gold slides) were loaded into a microarray printing robot (Bio-Rad VersArray Chipwriter) in which 3- $\mu \mathrm{M}$ antigens in PBS were printed using solid pins at $25{ }^{\circ} \mathrm{C}$ and $60 \%$ humidity, resulting in microarray feature diameters of $\sim 400 \mu \mathrm{m}$. The slides were dried in a desiccator and then blocked in PBST solution containing 5\% fetal bovine serum. Human serum was diluted into FBS solution (1:10), and $20 \mu \mathrm{l}$ of each solution was applied to each set of spots and incubated (shaking) for $1 \mathrm{~h}$ at room temperature, followed by washing twice with PBST and once with PBS. The array was then incubated in $1 \mathrm{nM}$ IRDye800-conjugated goat anti-human IgG (Vector Laboratories, Catalog \# AI-3080) in FBS for 20 min (shaking) at room temperature in the dark. Chips were washed twice with PBST and once with PBS, followed by immersion in deionized water and subsequent drying with compressed air. The total processing time to test the human samples for autoantibodies using the plasmonic gold chips was less than $2 \mathrm{~h}$.

\section{Fluorescence measurement and analysis}

A Licor Odyssey scanner was used to scan the IRDye800-secondary antibody-labeled microarrays on different substrates using the $800-\mathrm{nm}$ channel with the gain set to 6.0 as defined by the system and the resolution set to $42 \mu \mathrm{m}$. Microarray fluorescence images were 
analyzed by Genepix 6.1, and the spot features were automatically identified by the program. The fluorescence intensity of each spot was background corrected, and the average of the mean pixel intensity for each triplicate was calculated as the MFI of the feature. Note that the background signal was typically on the order of 10 , whereas the spot signals were in the range of 10-100,000 for various autoantibody concentrations.

\section{Calibration curves for insulin-, GAD- and IA2-specific antibodies}

For generating the calibration curves of each autoantibody, a series of standard samples with known amounts of autoantibody (Kronus) were applied to different microarrays on plasmonic chips, following the same procedure as described for human serum. Fluorescence signal versus autoantibody concentration was plotted and used for calibrating autoantibody concentrations in patient serum samples.

\section{Statistical analyses}

We measured the MFI for each subject against insulin, GAD65 and IA2 (ICA512) islet antigens on the plasmonic gold platform and directly compared these results to the known corresponding RIA data. Positive thresholds for the gold platform were determined by MFI measurements of control samples (obtained from the Kronus RIA kits) corresponding with the commercial Esoterix RIA positive threshold. We compared positive and negative MFI signals on our platform against RIA results to determine sensitivity and specificity for each diabetes autoantibody by receiver-operating characteristic plots. Using subsequent clinical diagnosis determined from clinical progression and insulin needs at six months after disease presentation, we assessed each subject's diabetes autoantibody profile against their clinical diagnosis to compare the sensitivity and specificity of our platform against RIA as a diabetes diagnostic tool. We used a positive signal in any of the three autoantibodies as diagnostic for T1D. As the autoantibody profile included three MFI values in non-normal distribution, we conducted quadratic discriminate analysis of the subject data. No statistical method was used to predetermine sample size. Subject recruitment was not randomized.

\section{Additional methods}

Detailed methodology is described in the Supplementary Methods.

\section{Supplementary Material}

Refer to Web version on PubMed Central for supplementary material.

\section{Acknowledgments}

We thank the patient volunteers. We thank P.J. Utz and D. Wilson for helpful discussions. We thank M. Gong, J. Wu and L. Zhang for help with experiments; we thank C. Yan for help with the scatter plot for diabetes autoantibodies. This work was supported by grants from the Stanford C-IDEA program (US National Institutes of Health grant 1 RC4 TW008781-01), the US National Institutes of Health DP2OD006740 (to B.J.F.) and the Juvenile Diabetes Research Foundation 17-2013-528 (to B.J.F.) and the National Cancer Institute of the US National Institutes of Health (5R01CA135109-02) and the Stanford SPARK program (to H.D.). B.Z. acknowledges support from the Stanford Bio-X SIGF fellowship. R.B.K. received unrestricted fellowship support from Genentech and the Child Health Research Institute at Stanford. B.J.F. is a Bechtel Endowed Faculty Scholar. 


\section{References}

1. Smyth S, Heron A. Diabetes and obesity: the twin epidemics. Nat. Med. 2006; 12:75-80. [PubMed: 16397575]

2. Karvonen M, et al. Incidence of childhood type 1 diabetes worldwide. Diabetes Mondiale (DiaMond) Project Group. Diabetes Care. 2000; 23:1516-1526. [PubMed: 11023146]

3. EURODIAB ACE Study Group. Variation and trends in incidence of childhood diabetes in Europe. Lancet. 2000; 355:873-876. [PubMed: 10752702]

4. International Diabetes Federation (IDF). Diabetes in children: epidemiology. Pediatr. Diabetes. 2007; 8(S8):10-18.

5. Patterson CC, et al. Incidence trends for childhood type 1 diabetes in Europe during 1989-2003 and predicted new cases 2005-20: a multicentre prospective registration study. Lancet. 2009; 373:20272033. [PubMed: 19481249]

6. Maahs DM, West NA, Lawrence JM, Mayer-Davis EJ. Epidemiology of type 1 diabetes. Endocrinol. Metab. Clin. North Am. 2010; 39:481-497. [PubMed: 20723815]

7. Liese AD, et al. The burden of diabetes mellitus among US youth: prevalence estimates from the SEARCH for Diabetes in Youth Study. Pediatrics. 2006; 118:1510-1518. [PubMed: 17015542]

8. Imperatore G, et al. Projections of type 1 and type 2 diabetes burden in U.S. population aged <20 years through 2050: dynamic modeling of incidence, mortality, and population growth. Diabetes Care. 2012; 35:2515-2520. [PubMed: 23173134]

9. Jones KL. Role of obesity in complicating and confusing the diagnosis and treatment of diabetes in children. Pediatrics. 2008; 121:361-368. [PubMed: 18245428]

10. Zeitler P. Approach to the obese adolescent with new-onset diabetes. J. Clin. Endocrinol. Metab. 2010; 95:5163-5170. [PubMed: 21131537]

11. Michels AW, Eisenbarth GS. Immune intervention in type 1 diabetes. Semin. Immunol. 2011; 23:214-219. [PubMed: 21852151]

12. Greenbaum CJ, Schatz DA, Haller MJ, Sanda S. Through the fog: recent clinical trials to preserve beta-cell function in type 1 diabetes. Diabetes. 2012; 61:1323-1330. [PubMed: 22618767]

13. Orban T, et al. Co-stimulation modulation with abatacept in patients with recent-onset type 1 diabetes: a randomised, double-blind, placebo-controlled trial. Lancet. 2011; 378:412-419. [PubMed: 21719096]

14. Greenbaum CJ, Palmer JP, Kuglin B, Kolb H. Insulin autoantibodies measured by radioimmunoassay methodology are more related to insulin-dependent diabetes mellitus than those measured by enzyme-linked immunosorbent assay: results of the Fourth International Workshop on the Standardization of Insulin Autoantibody Measurement. J. Clin. Endocrinol. Metab. 1992; 74:1040-1044. [PubMed: 1569152]

15. Liu E, Eisenbarth GS. Accepting clocks that tell time poorly: fluid-phase versus standard ELISA autoantibody assays. Clin. Immunol. 2007; 125:120-126. [PubMed: 17904423]

16. Valdez SN, Poskus E. Autoimmune diabetes mellitus: the importance of autoantibodies for disease prediction and diagnostic support. Curr. Immunol. Rev. 2010; 6:299-313.

17. Bottazzo GF, Florin-Christensen A, Doniach D. Islet-cell antibodies in diabetes mellitus with autoimmune polyendocrine deficiencies. Lancet. 1974; 304:1279-1283. [PubMed: 4139522]

18. Bingley PJ, Bonifacio E, Mueller PW. Diabetes Antibody Standardization Program: first assay proficiency evaluation. Diabetes. 2003; 52:1128-1136. [PubMed: 12716742]

19. Bingley PJ, et al. Measurement of islet cell antibodies in the Type 1 Diabetes Genetics Consortium: efforts to harmonize procedures among the laboratories. Clin. Trials. 2010; 7:S56S64. [PubMed: 20693189]

20. Schlosser M, Mueller PW, Torn C, Bonifacio E, Bingley PJ. Diabetes Antibody Standardization Program: evaluation of assays for insulin autoantibodies. Diabetologia. 2010; 53:2611-2620. [PubMed: 20871974]

21. Yu L, et al. Distinguishing persistent insulin autoantibodies with differential risk: nonradioactive bivalent proinsulin/insulin autoantibody assay. Diabetes. 2012; 61:179-186. [PubMed: 22124462] 
22. Törn C, et al. Diabetes Antibody Standardization Program: evaluation of assays for autoantibodies to glutamic acid decarboxylase and islet antigen-2. Diabetologia. 2008; 51:846-852. [PubMed: 18373080]

23. Tabakman SM, et al. Plasmonic substrates for multiplexed protein microarrays with femtomolar sensitivity and broad dynamic range. Nat. Commun. 2011; 2:466. [PubMed: 21915108]

24. Zhang B, et al. Multiplexed cytokine detection on plasmonic gold substrates with enhanced nearinfrared fluorescence. Nano Research. 2013; 6:113-120.

25. Achenbach $P$, et al. Combined testing of antibody titer and affinity improves insulin autoantibody measurement: Diabetes Antibody Standardization Program. Clin. Immunol. 2007; 122:85-90. [PubMed: 17059894]

26. Roberts MJ, Bentlye MD, Harris JM. Chemistry for peptide and protein PEGylation. Adv. Drug Deliv. Rev. 2002; 54:459-476. [PubMed: 12052709]

27. Oak S, Phan TT, Gilliam LK, Hirsch IB, Hampe CS. Animal insulin therapy induces a biased insulin antibody response that persists for years after introduction of human insulin. Acta Diabetol. 2010; 47:131-135. [PubMed: 19547910]

28. Naserke HE, Dozio N, Ziegler AG, Bonifacio E. Comparison of a novel micro-assay for insulin autoantibodies with the conventional radiobinding assay. Diabetologia. 1998; 41:681-683. [PubMed: 9662050]

29. Ljungberg UK, et al. The interaction between different domains of staphylococcal protein A and human polyclonal $\operatorname{IgG}, \operatorname{IgA}, \operatorname{IgM}$ and $\mathrm{F}\left(\mathrm{ab}^{\prime}\right)_{2}$ : separation of affinity from specificity. Mol. Immunol. 1993; 30:1279-1285. [PubMed: 8413328]

30. Wabl M, Cascalho M, Steinberg C. Hypermutation in antibody affinity maturation. Curr. Opin. Immunol. 1999; 11:186-189. [PubMed: 10322147]

31. Lakowicz JR. Radiative decay engineering 5: metal-enhanced fluorescence and plasmon emission. Anal. Biochem. 2005; 337:171-194. [PubMed: 15691498] 
a

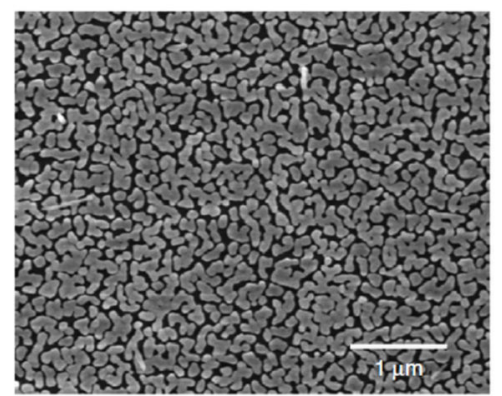

C

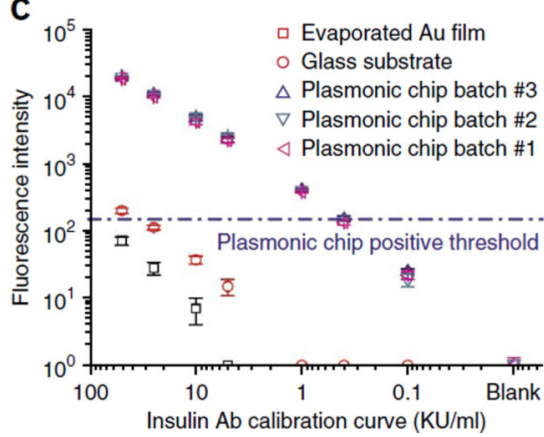

b


d
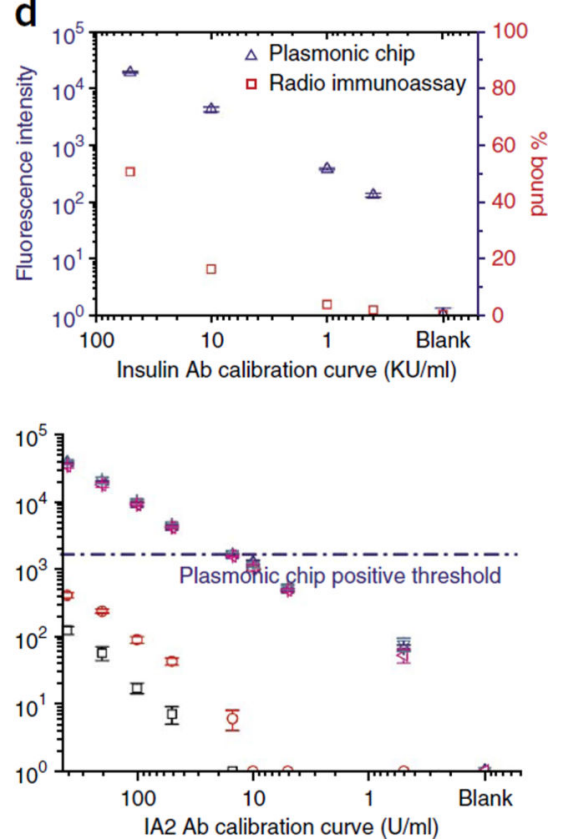

Figure 1.

Greater signal detection on plasmonic gold surface compared with standard surfaces. (a)

Electron micrograph demonstrating gold islands and abundant nanogaps in the nanostructured gold plasmonic film. (b) Schematic depicting the spatial relationship of the platform's PEG layer, the islet-specific antigens, the primary autoantibodies (Abs) from diluted human serum or blood and the detection antibodies conjugated with a fluorophore signal. (c) Calibration curves comparing detection limit and dynamic range of islet antigen autoantibody quantification on plasmonic gold, glass and evaporated gold substrates. Samples used for the calibration curves were standards containing known concentrations of autoantibodies in serum provided by the vendor (Kronus). Concentrations of standard samples for auto-insulin are in vendor's unit $(\mathrm{KU} / \mathrm{ml})$, and concentrations of standard samples for autoantibodies against GAD65 and IA2 are in the international unit of U/ml. We performed three independent experiments. Data shown as the mean \pm s.d. $(n=3)$. (d) Comparison of detection limits and dynamic ranges of insulin-specific autoantibody quantification between plasmonic gold and RIA. RIA data is from vendor-provided RIA kits. Data shown as the mean \pm s.d. $(n=3)$. 
a

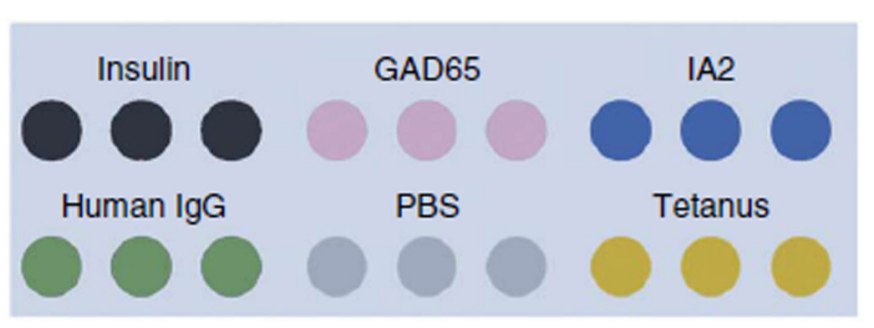

b

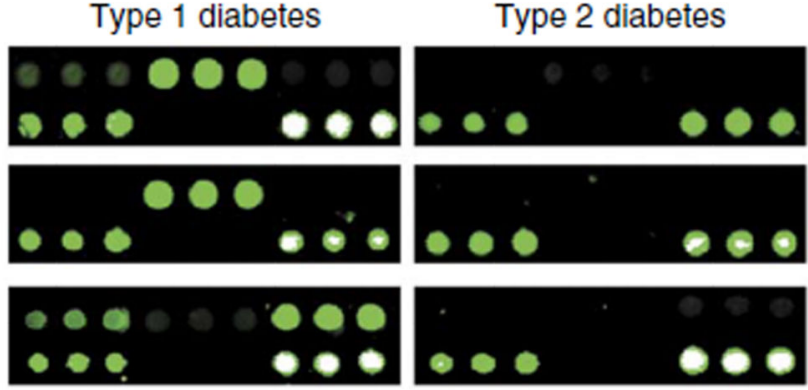

d


Insulin $\mathrm{Ab}$

GAD65 Ab

IA2 Ab
C

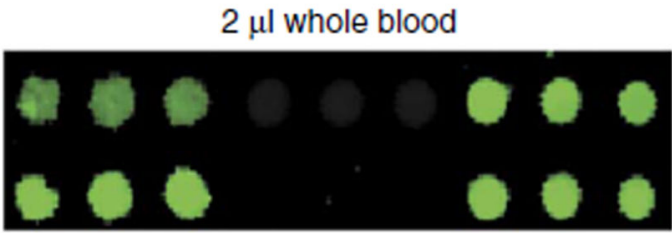

$2 \mu \mathrm{l} \mathrm{serum}$

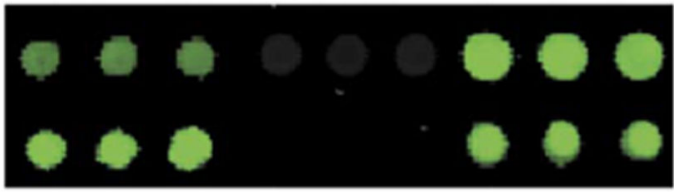

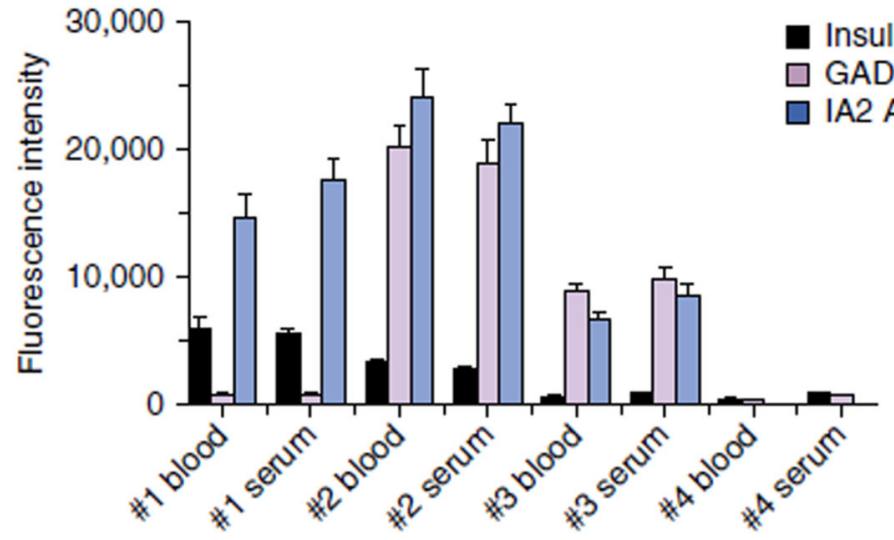

Figure 2.

The plasmonic chip readily differentiates T1D and T2D in ultralow serum or blood samples.

(a) Schematic layout of triplicate islet cell antigens, human IgG (positive control for the detection antibody), PBS (negative control) and tetanus toxoid (positive control for antibody detection in human serum) on the plasmonic gold chip. (b) Fluorescence mapping result (left) and signal quantification (right) on plasmonic gold chips for islet antigen-specific autoantibody detection of typical patients with T1D or T2D and nondiabetic controls. Error bars represent error between signals of the triplicate spots for each antigen shown in (b, left); we conducted the array experiments three times, as reflected in Supplementary Figure 6. (c) Comparison of signal on chips tested with whole blood or serum from a typical subject (left) and quantification of signals comparing whole blood and serum in four independent patients (right). We performed three independent experiments. Data shown as the mean \pm s.d. (d) Ultralow sample volumes can be used to profile diabetes autoantibodies in human serum or blood. 


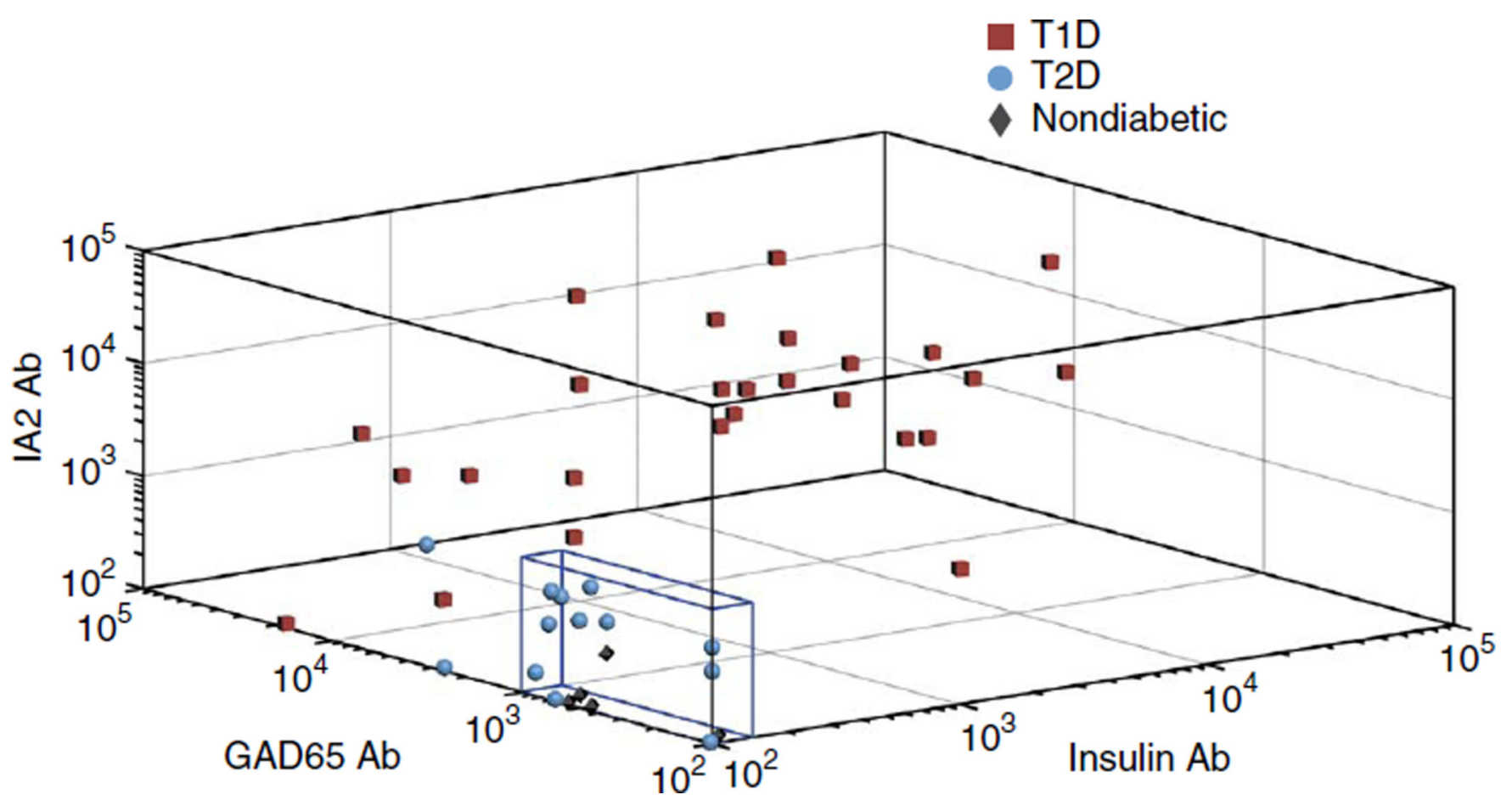

Figure 3.

Scatter plot for diabetes autoantibodies. Analysis of our subject pool of 26 children with new-onset T1D, 13 children with new-onset T2D and 5 nondiabetic children demonstrates a specific (i.e., non-normal) distribution of MFI values for the patients with T1D. Each point represents an individual patient. The titer for each of the three autoantibodies for the individual patient is plotted on the three-dimensional axes. Points that fall within the blue box are negative for T1D by plasmonic gold platform testing. Ab, antibody. 


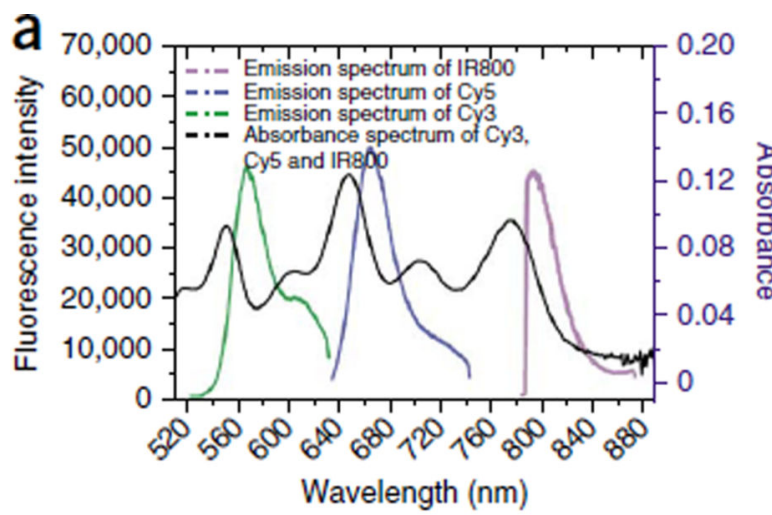

Wavelength $(\mathrm{nm})$

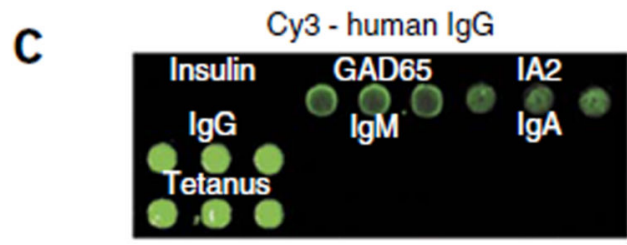

b

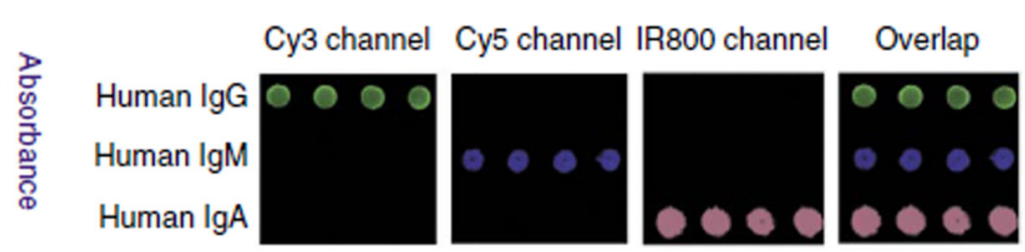

Figure 4.

The plasmonic chip permits differentiation of immunoglobulin isotypes from a single ultralow volume sample. (a) Absorbance and fluorescence emission of fluorophore Cy3, Cy5 and IRDye800. The emission spectra of the three fluorophores do not overlap with the emission spectrum at the trough of the absorbance spectrum (black), preventing absorption by fluorophores with adjacent emission spectra. (b) Simultaneous, specific detection of IgG, IgM and IgA isotypes on a multiplexed plasmonic gold chip using secondary antibodies with narrow, nonoverlapping emission spectra. (c) Multiplexed detection of islet cell-targeting autoantibody isotypes in a child with new-onset T1D. The multiplexed plasmonic gold chip simultaneously detects specific IgG autoantibodies against GAD65 and IA2, as well as IgM autoantibodies against insulin and GAD65, from a single sample from the example patient. 
Table 1

Comparison of patient test results by RIA and gold plasmonic platforms

\begin{tabular}{lcc}
\hline & RIA & Gold plasmonic $^{\boldsymbol{a}}$ \\
\hline T1D sensitivity & $\mathbf{1 0 0 \%}$ & $\mathbf{1 0 0 \%}$ \\
T1D specificity & $\mathbf{8 5 \%}$ & $\mathbf{8 5 \%}$ \\
Number of patients with: & & \\
Zero antibodies positive & 11 & 11 \\
One antibody positive & 7 & 5 \\
Two antibodies positive & 7 & 9 \\
Three antibodies positive & 14 & 14 \\
\hline
\end{tabular}

${ }^{a}$ Positive cutoff equivalence: insulin $=0.4 \mathrm{KU} / \mathrm{ml}, \mathrm{GAD} 65=5 \mathrm{U} / \mathrm{ml}$ and IA2 $=15 \mathrm{U} / \mathrm{ml}$. Patients with one or more positive autoantibody were considered positive for T1D by that platform. 